\title{
Combined airborne thermography and visible-to-near infrared reflectance measurement for soil moisture mapping
}

\author{
by J.-C. Krapez*, C. Chatelard*, J.-F. Nouvel** and Ph. Déliot***
}

\author{
*ONERA, The French Aerospace Lab, DOTA, F-13661 Salon de Provence (France), krapez@onera.fr \\ ${ }^{* *}$ ONERA, The French Aerospace Lab, DEMR, F-13661 Salon de Provence (France) \\ ${ }^{* * *}$ ONERA, The French Aerospace Lab, DOTA, F-31055 Toulouse (France)
}

\begin{abstract}
We first present a review of the so-called triangle/trapezoid method for soil moisture evaluation from optical remote sensing data obtained in the red, near infrared and thermal infrared bands. Recent improvements based on additional vegetation indexes obtained for example from multiwavelength signals in SWIR band will also be presented. In a second part we describe the results we obtained for this purpose by using a microbolometer camera onboard of a motorglider. Signal processing for atmosphere correction and orthorectification was specifically developed for this application. First results on the path for identification of soil water content with this approach will be presented.
\end{abstract}

\section{Introduction - A review of thermal remote sensing methods for soil water content assessment}

\subsection{Soil moisture monitoring}

Soil moisture is one of the key variables in the hydrologic cycle controlling processes such as infiltration and discharge with consequences for surface water availability and plant growth. It also plays an important role in the exchange of mass and energy in the soil-plant-atmosphere system through evaporation and transpiration. Information on soil moisture is critical in agricultural water management: assessment of spatial distribution of soil water content at field scale is needed for evaluating crop water requirement, optimizing the irrigation procedures and improving productivity. In hydrology, estimation of soil moisture at watershed scale is critical for predicting natural disasters, such as flooding and droughts. For this reason, monitoring of soil moisture with high spatial coverage, high temporal frequency and a range of resolution is highly desirable.

Conventional soil moisture observations are often inadequate: thermo-gravimetric and TDR/FDR (Time/ Frequency domain reflectometry) measurements are time consuming and labour expensive. Furthermore they only provide very local data. The consequence of this scarcity is a low representativeness of the retrieved information.

Remote sensing has the ability to collect information from contiguous samples at large scale in a short time and repeated time intervals thus allowing the generation of soil moisture maps at different spatial scales and resolutions.

Techniques based on active and passive microwave remote sensing have been developed for a few decades. They offer the advantage of not being affected by the weather conditions (low sensitivity to cloud cover). They however present some specific limitations: the satellite type passive sensors are characterized by a low spatial resolution, about $50 \mathrm{~km}$, whereas active microwave like synthetic aperture radar is dependant on vegetation cover and surface roughness which requires a priori information.

Optical remote sensing techniques offer the advantage of a higher spatial resolution. The reflective (visible to near/mid-infrared) and the thermal spectral domains were both considered for the following reasons.

The first one is that changes in soil water content lead to variations of the spectral reflectance. Also, water present in the root zone has a direct impact on the physiological state of a plant, thus modifying the spectral characteristics of a leaf and affecting the reflectance of the canopy. However applications for the determination of soil moisture were generally limited to bare soil surfaces; they also face the problem that variations of reflectance with soil moisture and with soil organic content are similar, thus requiring additional information.

The second reason is that temperature of both soil surface and vegetation is an indicator of water availability. This thermal signature is most clearly revealed near midday when the solar heating flux is highest. Some satellite applications also considered the temperature minimum reached during night: the product obtained by dividing the absorptance in the solar spectral domain by the temperature difference between day and night was named apparent thermal inertia. Nevertheless, temperature contrasts at night are generally very low, the highest informative content is found in the midday temperature map. The energy balance for soil can be expressed as:

$$
R_{n}=G+\lambda E+H
$$

where $R_{n}, G, \lambda E$ and $H\left(\mathrm{~W} / \mathrm{m}^{2}\right)$ are net radiation, soil heat flux, latent heat flux and sensible heat flux, respectively. Evaporation at the soil surface limits its temperature increase. Thus the soil surface temperature is inversely correlated with water content. Additionally, soil wetting induces an increase of soil thermal effusivity which limits 
temperature excursion between night and day. Through its influence on both evaporation and thermal effusivity, a soil moisture increase thus leads to a reduction of midday surface temperature. On the other hand, the soil moisture influence on vegetation temperature is as follows: under proper water supply, the leaf transpiration prevents its temperature from rising too high above air temperature. On the opposite, under water stress condition, the stomata on the leaf surface close to limit water loss. This transpiration reduction leads to a temperature increase of the foliage.

However, the soil surface temperature and the plant temperature are not influenced by the same portion of the moisture depth profile. Evaporation at the soil surface depends on the moisture present in the first centimetres. The heat diffusion depth corresponding to a $24 \mathrm{~h}$ cycle is larger, namely a couple of decimetres, however the moisture profile has a vanishingly influence with depth on the surface temperature. By contrast, leaf temperature is monitored by the moisture present in layer which thickness is of the order of one meter for crops: the root zone layer. In addition, the reaction of root zone moisture on leaf temperature presents a time lag. Altogether we can state that soil surface temperature and canopy temperature both provide information on soil moisture but at different depth scale and at different time scale. A specific problem appears when the measurement is a mixture of soil temperature and vegetation temperature. As a matter of fact airborne or satellite thermal sensors used for environment monitoring have a spatial resolution ranging from a few meters to a couple of kilometres. The consequence is that in the footprint of a pixel, the surface is seldom homogenous: the infrared radiation reaching most elementary detectors is a combination of the radiance coming from leaves and from bare soil. As a first consequence, the measured brightness temperature depends on temperature and emissivity of both soil and vegetation through the vegetation cover fraction. Part for this reason, the problem of evaluating soil moisture is underdetermined when one only considers the brightness temperature. There are basically three unknown variables: cover fraction, soil temperature and vegetation temperature (at present, we consider that vegetation is of one type only, at the same stage of maturity). From the second variable one can expect to evaluate the soil moisture in a shallow layer a few centimetres thick, from the third variable one can expect to evaluate the soil moisture in the root zone. Most works partly reduce the underdetermination by considering just one additional observation data. The consequence is that the inferred moisture information is a complex function of the soil moisture depth profile which is actually never presented. This is why the inferred variable is sometimes simply called moisture availability.

\subsection{Soil moisture evaluation from 2D scatterplot of temperature vs. vegetation index}

The additional observation data is a vegetation index (VI) used as a proxy of the vegetation cover [1-5]. It is generally the well known NDVI (Normalized Difference Vegetation Index) which corresponds to the normalized difference between the signals recorded in the red and in the near infrared (typically at about $0.65 \mu \mathrm{m}$ and $0.8 \mu \mathrm{m}$ ). This index is indeed strongly correlated to the vegetation cover: it ranges from about 0.1 for bare soil to values close to 0.8 for dense and green vegetation. It is also correlated to foliage density which is expressed by LAI, Leaf Area Index (total surface of leaves per surface unit). However NDVI also slightly depends on the soil spectral characteristics; for this reason other indexes less dependant on soil background were proposed like TSAVI (Transformed Soil Adjusted Vegetation Index) and OSAVI (Optimized Soil Adjusted Vegetation Index) [6].

When plotting the two-dimensional distribution of temperature and vegetation index (T-VI) corresponding to an area with well distributed vegetation cover and moisture content, one gets a scatter of triangular of trapezoidal shape (see figure 1). The four vertices correspond to the extreme conditions of a crop [1]: the bottom vertices (low VI) correspond to dry and saturated bare soil, whereas the upper vertices (high VI) correspond to well-irrigated and waterstressed full cover vegetation. The left limit is called the "wet" edge (or "cold" edge) whereas the right limit is called the "dry" edge (or "warm" edge).

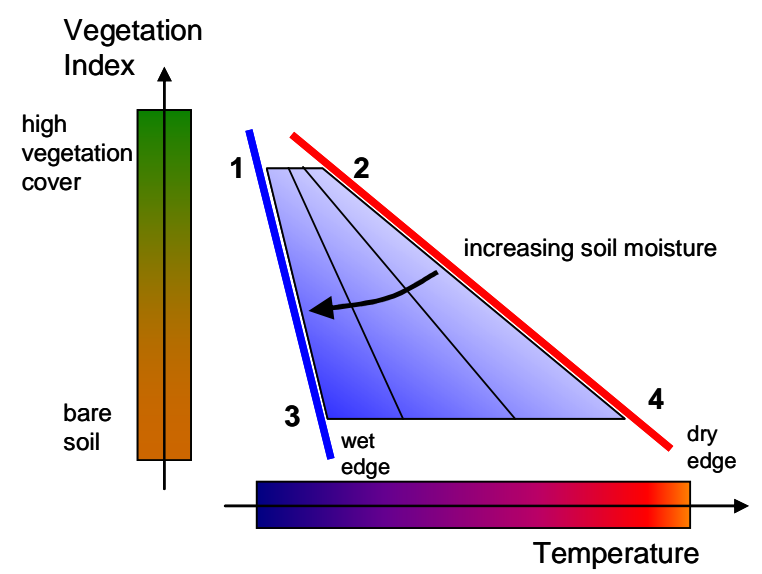

Fig. 1. Principle of soil moisture mapping from a Temperature-Vegetation Index (T-VI) plot [1]. The vertices of the trapezoidal distribution correspond to: 1 : well-watered full cover, 2 : non-transpiring full cover, $3:$ wet bare soil, 4 : dry bare soil. Soil moisture increases from the dry edge (on the right) to the wet edge (on the left)

Different methods were devised for setting the wet and dry edges. 
The method of Moran et al. [1] is based on the use of the Penman-Monteith equation for evapotranspiration. It allows expressing the difference between surface temperature and air temperature for the limiting cases corresponding to the four vertices of the trapezoidal T-VI plot (see fig. 1). It is admitted that the soil dryness linearly increases when moving from the wet edge to the dry edge [1,4]. For this reason, a soil moisture index (the Water Availability Index WAI) can be defined from the relative distance, at constant $\mathrm{VI}$, of a given point to the dry edge [5] (WAI = 1-WDI, where WDI is the Water Deficit Index defined in [1]). Similarly, it is argued that the vegetation stress increases from the wet edge to the dry edge [1]. A consequence is that the position of a given point relatively to these border lines could also be a measure of the evapotranspiration flux at the time of the remote sensing test. Iso-moisture straight lines are then distributed linearly between the wet edge and the dry edge. Based on a statistical approach for the evaluation of the trapezoid vertices position, plots of the mean WAI values in the T-NDVI space and of the corresponding standard error were built in [5] (see fig. 2). The right plot shows in particular that the precision in evaluating the Water Availability Index worsens for rising NDVI, i.e. high vegetation cover fraction. Sensitivity of thermal remote sensing to soil moisture thus decreases in presence of dense vegetation.
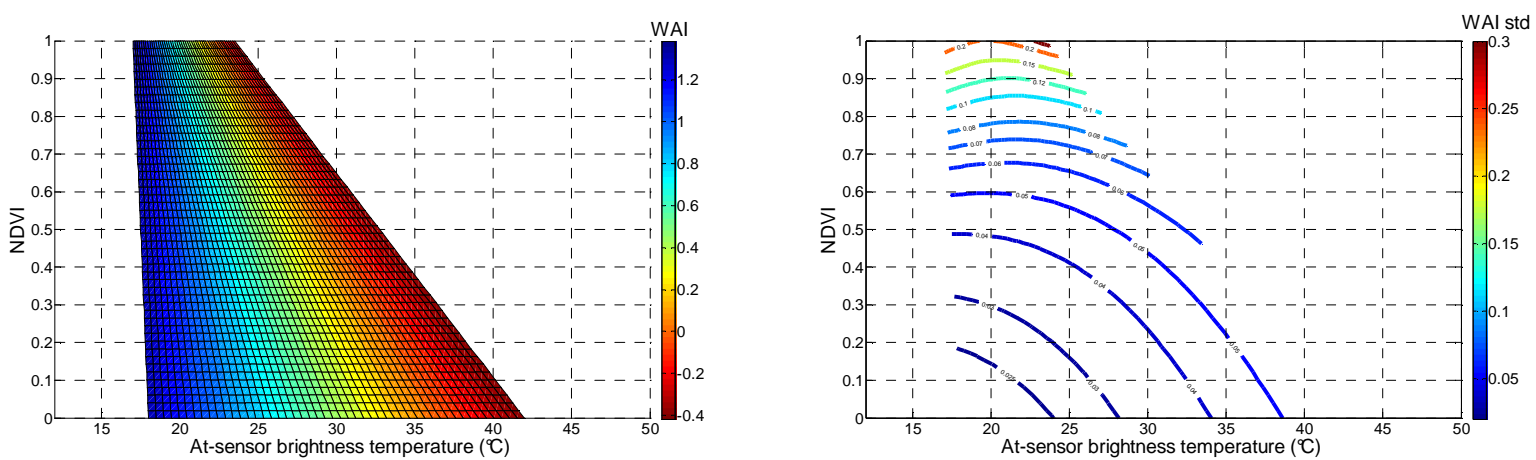

Fig. 2. Left : Colormap of mean Water Availability Index (WAI) in the T-NDVI space. Right : Isolevel curves describing the WAI standard error in the T-NDVI space (from [5]).

The method of Carlson et al. [2, 3] is based on the use of a SVAT model (Soil Vegetation Atmosphere Transfer). This model allows evaluating the radiation flux, the convective flux and the water/vapor fluxes between soil, vegetation and atmosphere. For a given soil moisture content, it thus enables to calculate the brightness temperature averaged over soil and vegetation for the time of the acquisition. Iso-moisture lines, which are not necessarily straight lines, can then be drawn. These isoplets can be interpolated with a bivariate polynomial to get a practical inversion tool [2, 3]. Simulations were performed in [5] with SEtHyS model by assuming different values for the root zone mean water content (see fig. 3). The results highlighted the fact that the temperature sensitivity to the upper soil layer moisture is most important at low NDVI (i.e. low vegetation cover fraction) whereas at high NDVI temperature is essentially sensitive to root zone moisture.

The methods presented so far rely on a preliminary calculation of the iso-moisture lines, let them be straight lines, like in the Moran method [1], or curved lines, like in the Carlson method [2, 3]. The temperature calculation requires a substantial number of parameters, particularly when using a SVAT model (meteorological data, soil and vegetation properties...). In addition, due to the estimation error of most parameters, the T-NDVI experimental scatterplot rarely matches with the theoretical one. A normalizing/stretching procedure is therefore often required for them to fit together and for allowing soil moisture identification [3]. This normalizing/stretching procedure relaxes the requirement for precise data about radiative fluxes and atmospheric fluxes and about soil/vegetation intrinsic properties, it however opens the way to a simpler approach which is the empirical method proposed by Sandholt et al. [4].
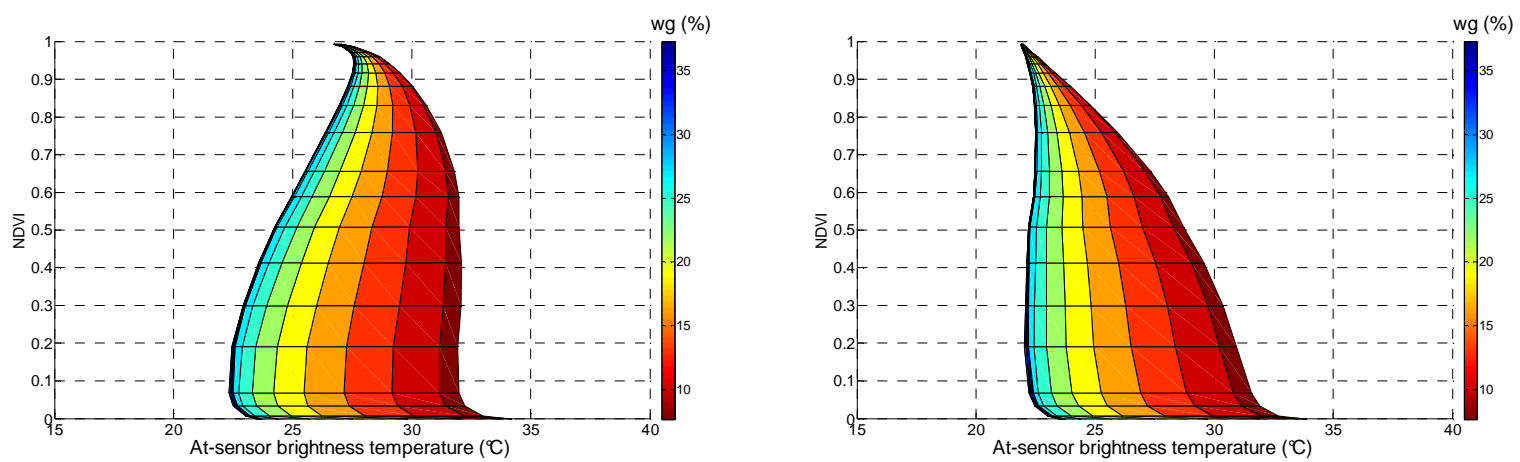

Fig. 3. Temperature versus NDVI and upper soil layer water content (wg \%vol.). Root zone mean water content was considered constant for each plot: $10 \% \mathrm{vol}$. (left) and $20 \% \mathrm{vol}$. (right). (from [5]). 
Sandholt's method in [4] is based on the experimental T-VI distribution only; no temperature computation is required. Two linear regressions are simply performed for fitting the low temperature border and the high temperature border of the scatterplot. The iso-moisture lines, assumed to be straight, are then evenly, i.e. linearly distributed between the wet edge and the dry edge. A large number of applications can now be found in the literature, they mainly deal with low resolution data, i.e. at field scale and landscape scale, as provided by satellite based sensors. The applicability to high resolution and multitemporal data was recently discussed [7].

The drawing of the lines defining the wet and dry edges as described in [4] is rather subjective. A more systematic and objective approach based on statistics was described in [8]: for each VI interval of width 0.05, a low temperature limit and a high temperature limit are defined from the p percentile and the 100-p percentile (typically $p$ is between $0.5 \%$ and $1.5 \%$ ). The results are then smoothed with cubic splines to provide the wet and dry edges as reported in fig. 4. By this way the scatterplot is tightly bounded; in the same time, possible outliers are rejected. At each Vegetation Index level, the Soil Vegetation Wetness Index (SVWI) is defined as the relative distance to the dry edge:

$$
S V W I_{i j}=\frac{T_{i j}-T_{d r y}\left(V I_{i j}\right)}{T_{w e t}\left(V I_{i j}\right)-T_{d r y}\left(V I_{i j}\right)}
$$

Other methods for rejecting the outliers can be found in $[2,7,9,10]$.

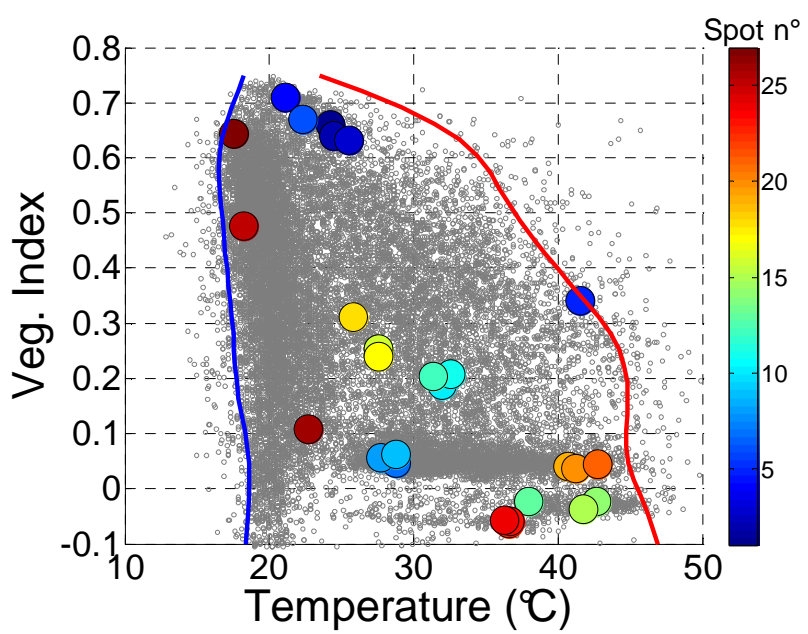

Fig. 4. Temperature-Vegetation Index scatterplot obtained during HyEurope 2007 campaign (from [8]). The wet edge (in blue) and the dry edge (in red) are built from $1 \%$ and $99 \%$ percentile temperature values for varying vegetation index. The dots with scaled color correspond to different spots/fields and crops.

\subsection{Addition of a third optical parameter to T-VI}

The works described so far use only two observation variables, namely temperature and a vegetation index. They rely on an over-simplified representation of the heat and mass transfers between the elements of the complex soil/vegetation/ atmosphere system. A large number of parameters, in addition to soil moisture, actually have an impact on the apparent soil/canopy temperature. As these parameters may vary between the different vegetation types and soil types, it is expected that moisture cannot be unambiguously retrieved from a given couple of T-VI values. The inversion cannot lead to a unique solution. To solve this problem one has to consider additional observations.

Chauhan et al. [11] suggested adding surface albedo. When introducing the albedo, it is expected to separate areas according to the absorbed solar radiation. Albedo can be evaluated with a good precision from the reflectance measurement in a limited number of spectral channels in the solar spectrum. Recently we looked for separating vegetated areas according to another property, namely the fraction of green vegetation versus senescent vegetation [8]. A suitable index for quantifying the fraction of senescent vegetation is CAI (Cellulose Absorption Index). It is computed from the reflectance measured in three bands: one at $2.1 \mu \mathrm{m}$, the cellulose-lignin absorption maximum, and two at the shoulders of this absorption band, at $2 \mu \mathrm{m}$ and $2.2 \mu \mathrm{m}$ :

$$
C A I=0.5\left(\rho_{2.0}+\rho_{2.2}-2 \rho_{2.1}\right)
$$

where $\rho_{i}$ is reflectance at wavelength $i$. Two examples of 3D scatterplots with respectively albedo and CAI as the third parameter are reported in fig. 5 . The boundary surfaces corresponding to the dry border, respectively the wet border, are again built from the $\mathrm{p} \%$ percentile and the $100-\mathrm{p} \%$ percentile temperature values. 

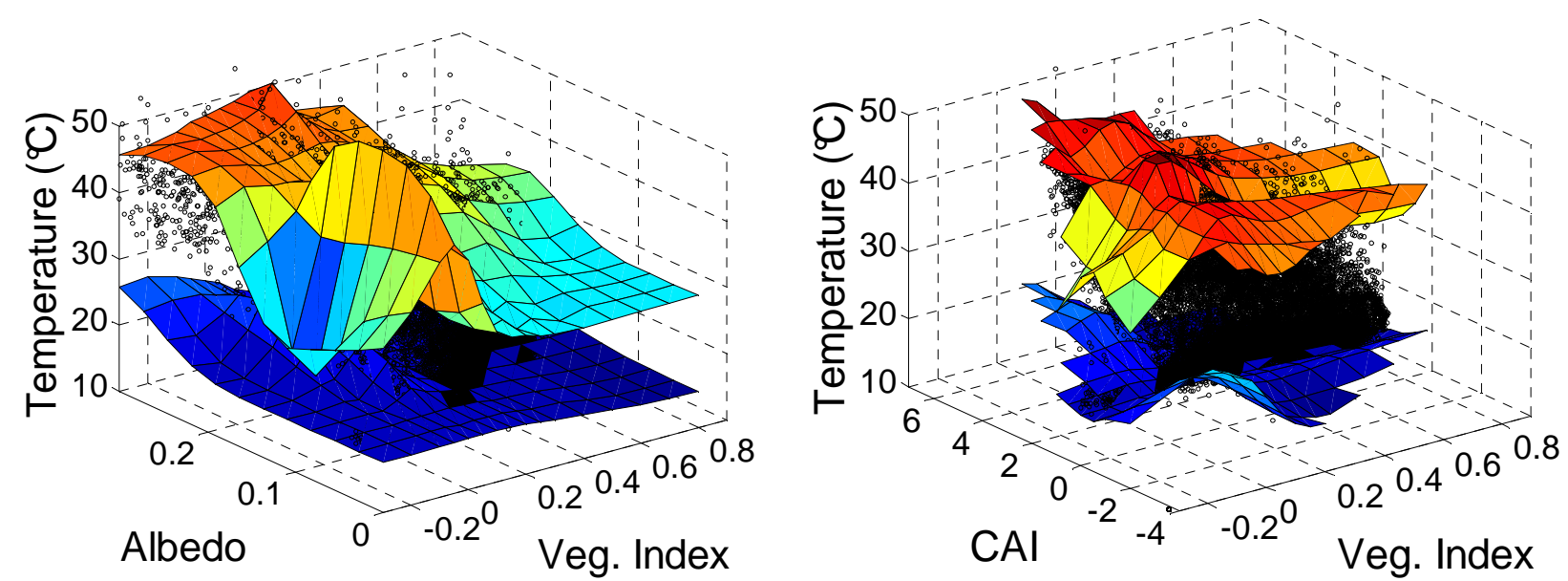

Fig. 5. Distribution of temperature, Vegetation Index and repectively albedo (left) and Cellulose Absorption Index (right). Each distribution is bounded on the hot side and on the cold side by the "dry", respectively the "wet" boundary surfaces (from [8]).

Introducing albedo didn't seem to provide an improvement in [8], whereas the addition of the Cellulose Absorption Index (CAl) showed to be more promising, indeed the correlation coefficient $\mathrm{R}^{2}$ between Soil Vegetation Wetness Index and the gravimetric moisture content increased from 0.61 to 0.69 . Nevertheless, the drawback of the proposed statistical approach for defining the wetness index is that the scatterplot must be well populated, i.e. it should present both dry soil and wet soil areas, for each couple of considered albedo-VI or CAI-VI values in the gridded plane. This is not always the case for a region of small extend.

Another additional parameter was recently considered: the infrared emissivity [12]. Soil moisture $M$ was evaluated from a relation proposed by Chauhan in [11] where albedo is replaced by the emissivity $\varepsilon$ :

$$
M=\sum_{i=0}^{2} \sum_{j=0}^{2} \sum_{k=0}^{2} a_{i j k} T^{*^{i}} N D V I *^{j} \varepsilon^{*^{k}}
$$

The coefficients $a_{i j k}$ of this multivariate polynomial regression were determined through a calibration process based on a series of ground moisture measurements. However the improvement by including the emissivity proved to be only slight when applied to remote sensing data.

\subsection{Present work}

Previous works on soil moisture evaluation by applying the T-VI method always used sophisticated and expensive equipment for mapping temperature and the vegetation index. Multispectral or even hyperspectral cameras of either pushbroom or whiskbroom technology were involved. An exception can be found in [7] where a broadband LWIR infrared camera was used. Our purpose was to evaluate the possibility of using a thermographic camera of microbolometer type on board of a motor-glider for soil moisture evaluation by the T-VI approach. The vegetation index itself was provided by a hyperspectral camera in the visible-NIR range. The use of a hyperspectral camera in this spectral range is justified because it proved to be extremely useful for soil classification applications. On the contrary, a multi/hyperspectral in the thermal domain is not absolutely necessary for soil moisture application. As a matter of fact, the motivation of a precise emissivity-temperature separation, which indeed would require a multi/hyperspectral approach, is not justified. On one hand emissivity of natural surfaces is generally high and uniform (see §3) and on the other hand, the triangle method can accept temperature relative values instead of absolute values. Furthermore temperature relative values can sometimes be considered independently of the vegetation cover fraction (see fig. 4 and fig. 5).

In a first part we will present the thermographic means and the processing for atmospheric correction. In a second part we will present the processing for image geometric correction and for geolocation.

\section{ENVIRO 2011 campaign}

A multisensor remote sensing campaign (radar + optic) was performed in October 2011 over an agricultural area near Garons-Nîmes, south of France. Two optical cameras were implemented: a hyperspectral camera in the visible to near infrared range (Hyspex VNIR1600 from NEO) and a LWIR microbolometer camera (A325 from FLIR), see tab. 1. 
The Hyspex camera was flown onboard a Falcon 20 on October $5^{\text {th }}$ and $6^{\text {th }}$. The FLIR A325 camera was flown onboard a STEMME S10-VT motorglider on October $21^{\text {st }}$ (see fig 6). The motorglider was equipped with a GPS receiver (Novatel OEM4-G2 card) in the cabin and with an Inertial Measurement Unit (iMAR iVRU-FAS-C167) in the left pod. GPS data and data from IMU were recorded during all the flight. Then, they were processed at ground level and combined in order to make up a precise plane trajectory record (see fig. 6-right). The trajectory and the attitude angles (heading, pitch, roll) were later used for orthorectifying and geolocating the infrared images.

The region of interest was covered by the motorglider with five overlapping flight lines. Mean altitude was 1090 $\mathrm{m}$, whereas ground mean altitude is $70 \mathrm{~m}$. Mean speed was $41 \mathrm{~m} / \mathrm{s}$ and an infrared image was recorded every $0.83 \mathrm{~s}$ thus insuring an overlapping of about $90 \%$.

\begin{tabular}{|l|l|l|}
\hline Instrument & \multicolumn{1}{|c|}{ Hyspex VNIR 1600 } & \multicolumn{1}{c|}{ A325 } \\
\hline FOV & $34^{\circ}$ (with FOV expander) & $25 \times 19^{\circ}$ \\
\hline Nb pixels & 1600 (Push-broom) & $320 \times 240$ \\
\hline Spectral range & $0.4-1 \mu \mathrm{m}$ & $7.5-13 \mu \mathrm{m}$ \\
\hline Nb. bands & 160 & 1 \\
\hline Spectral resolution & $3.7 \mathrm{~nm}$ & - \\
\hline Footprint & $0.75 \mathrm{~m} @ 2000 \mathrm{~m} \perp$ flight dir. & \multirow{2}{*}{$1.4 \mathrm{~m} @ 1020 \mathrm{~m}$} \\
\cline { 2 - 3 } & $1.50 \mathrm{~m} @ 2000 \mathrm{~m} / /$ flight dir. & \multicolumn{1}{|c|}{$450 \mathrm{~m} @ 1020 \mathrm{~m}$} \\
\hline Swath & $1200 \mathrm{~m} @ 2000 \mathrm{~m}$ & \\
\hline
\end{tabular}

Tab. 1. Technical characteristics of the cameras used during the Enviro 2011 campaign
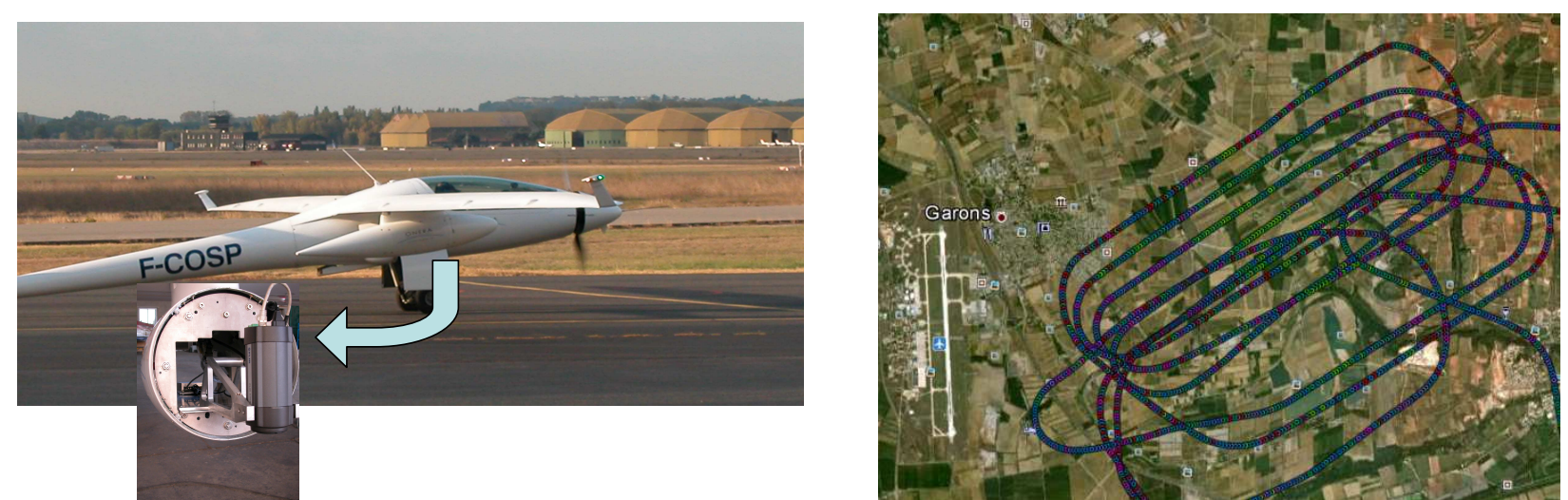

Fig. 6. Left : camera A325 installed in the right pod (shown opened in the small view) of the STEMME motorglider. Right: View of the five flight lines over the Garons area (mean coordinates: 4346'N, 42 7'E).

During the October $5^{\text {th }}$ flight an on-site analysis was performed for getting a precise classification of the vegetation (type, size, orientation...). Spectral reflectance of a series of leaf and soil samples was also measured on-site (ASD NIR FieldSpec). During the October $21^{\text {st }}$ flight, soil moisture was measured in 16 different fields with a FDR probe (SM200, Delta-T).

\section{Atmosphere correction of the infrared signal}

The A325 camera was set to provide brightness temperature images of the ground (blackbody equivalent temperature). It aimed at nadir through a hole in the pod. The spectral response of the camera is shown in fig. 7-left.

For performing the atmosphere correction we had to consider the average emissivity of the ground. Fig. 7-right reports the spectral emissivity of a series of vegetation types as found in the ASTER database [13]. The mean value in the spectral band of the camera is about 0.96 . A very close value was also obtained by averaging the data of saturated and dry soil samples extracted from six nearby fields $(<18 \mathrm{~km}$ from Garons test area) which were the object of a previous campaign [8]. For the remaining we will consider a common value of 0.96 , having nevertheless in mind that the mean emissivity could change by $+/-0.02$ depending on the vegetation type and water stress state.

The atmosphere correction was performed with MATISSE v2.0 software [14]. MATISSE (Advanced Modelling of the Earth for Environment and Scenes Simulation) is a radiative transfer code which provides spectral images of natural backgrounds, path transmission and radiance along a line of sight, sky radiance or local illumination around a target point. Natural backgrounds include clear atmosphere, water and ice clouds, geolocated land and sea. Its bandwidth ranges from 0.4 to $15 \mu \mathrm{m}$.

Radiosounding data measured at Nîmes-Courbessac, less than $12 \mathrm{~km}$ from the campaign area, provided the temperature and humidity profiles necessary for the MATISSE computations. The radiosounding profiles ranged from ground to $8 \mathrm{~km}$ altitude, it was thus necessary to extrapolate them with a standard profile, namely the MidLatitude Summer profile. The resulting temperature and water vapour content profiles are presented in fig. 8. 

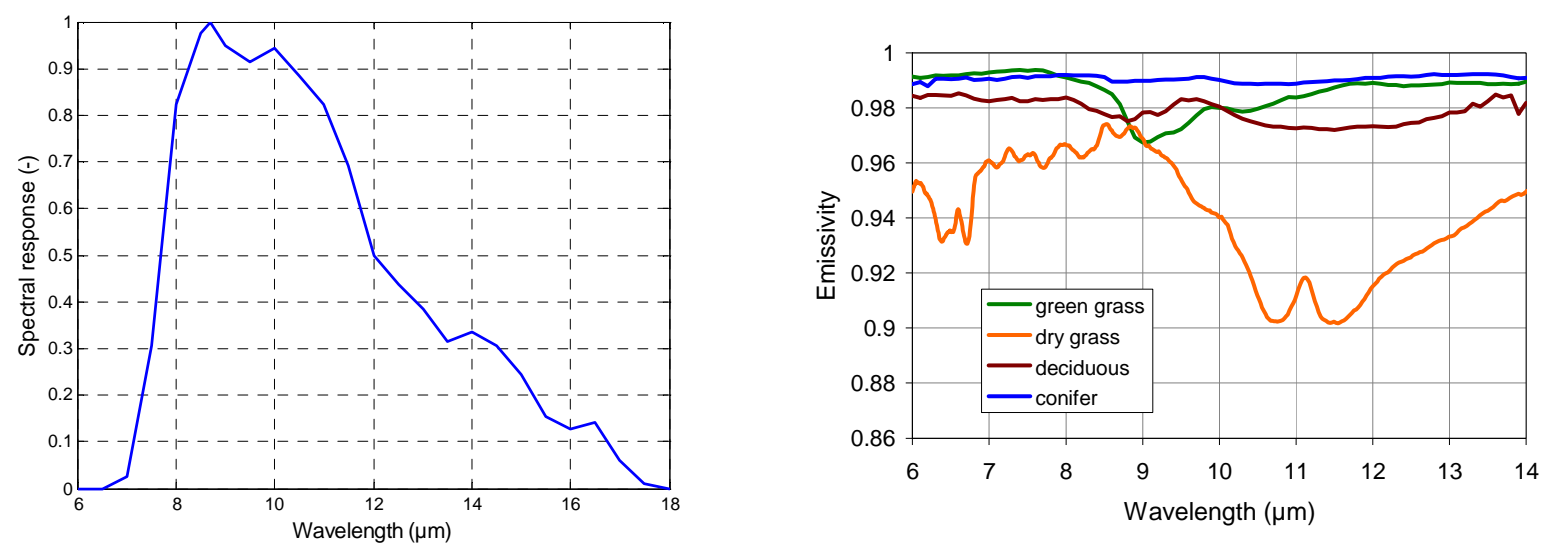

Fig. 7. Left: spectral response of the microbolometer camera A325 with a $25^{\circ}$ FOV optic (FLIR data). Right: emissivity of vegetation from the ASTER spectral library [13].

An example of spectral transmission and radiance computed with MATISSE is provided in fig. 9. It corresponds to nadir viewing at $1090 \mathrm{~m}$ altitude towards soil at $70 \mathrm{~m}$ altitude, assumed greybody $(\varepsilon=0.96, \mathrm{~T}=290 \mathrm{~K})$.
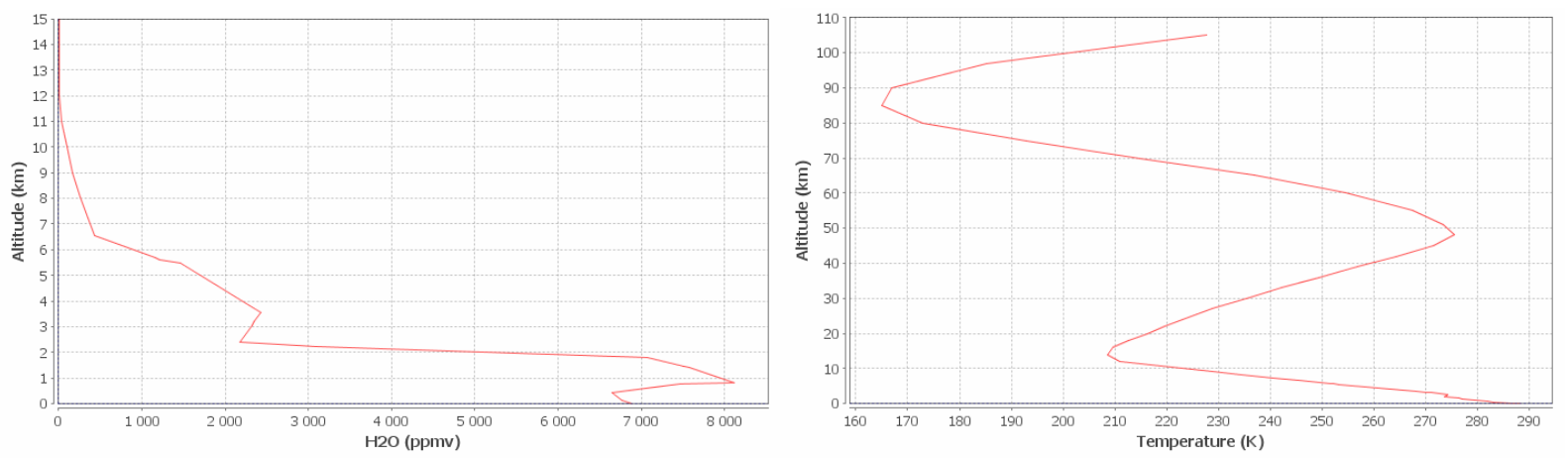

Fig. 8. Left : water profile, right : temperature profile obtained by merging radiosounding profiles and MidLatitude Summer profiles.
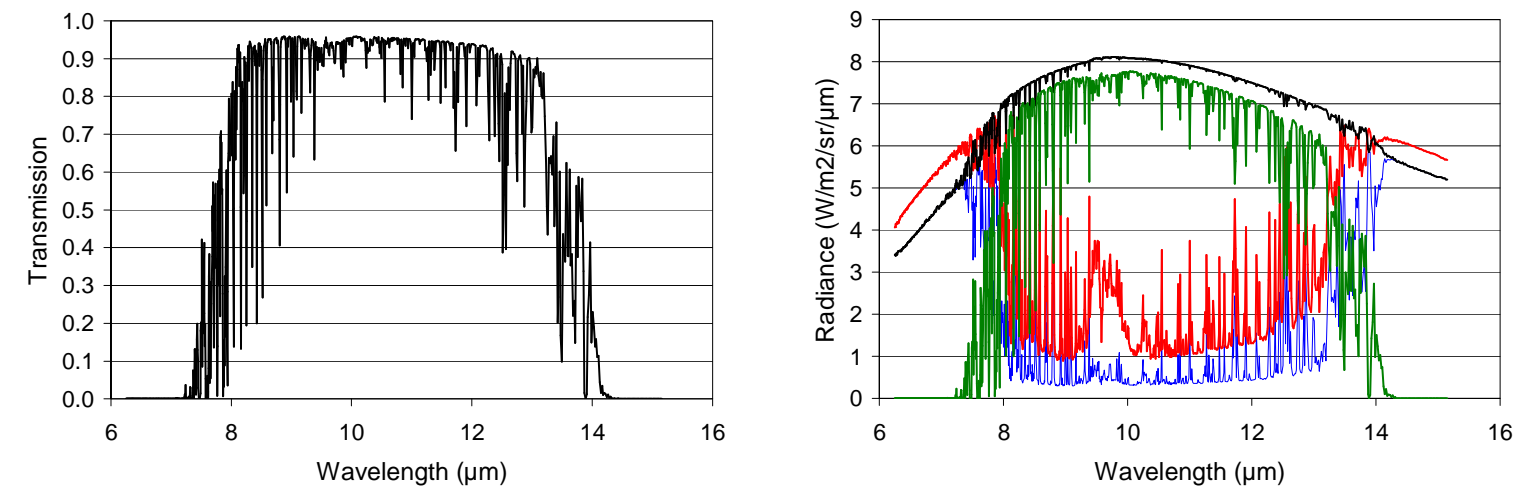

Fig. 9. Left: path transmission. Right: green curve: transmitted ground radiance (emitted + reflected by ground), blue : upwelling atmospheric radiance; black : total at-sensor radiance; red : downwelling sky radiance at ground level.

The radiative equations on which the temperature correction is based are [15]:

$$
\left\{\begin{array}{l}
L_{s}(\lambda, T, \theta)=\tau(\lambda, \theta)\left[\varepsilon(\lambda) B(\lambda, T)+(1-\varepsilon(\lambda)) \frac{E^{\downarrow}(\lambda)}{\pi}\right]+L^{\uparrow}(\lambda, \theta) \\
\int_{L W I R} S(\lambda) L_{s}(\lambda, T, \theta) d \lambda=\int_{L W I R} S(\lambda) B\left(\lambda, T_{a p p}\right) d \lambda
\end{array}\right.
$$


where $L_{s}(\lambda, T, \theta)$ is measured radiance at the sensor level in a direction of zenith angle $\theta$ (we assume azimutal symmetry), $B(\lambda, T)$ is blackbody radiance, $\tau(\lambda, \theta)$ is the path transmission, $E^{\downarrow}(\lambda)$ the downwelling sky irradiance $\left(L^{\downarrow}(\lambda)=E^{\downarrow}(\lambda) / \pi\right.$ is the corresponding isotropic equivalent radiance), $L^{\uparrow}(\lambda, \theta)$ is the upwelling path radiance emitted by the atmosphere constituents, $S(\lambda)$ is the LWIR camera spectral response, $T_{a p p}$ is the brightness temperature provided by the camera.

The link between brightness temperature and real ground temperature is described in fig. 10-left for different sensor altitudes and viewing angles. The ground is at $70 \mathrm{~m}$ altitude with a constant emissivity of 0.96 . Path transmission and radiance induce a temperature underestimation when temperature is higher than $3^{\circ} \mathrm{C}$. This underestim ation rises with temperature; it depends both on sensor altitude and on viewing angle.

Each curve in fig. 10-left was fitted with a degree-3 polynomial. The coefficients themselves were afterwards fitted with a linear function of height $h$ and distance $h / \cos \theta$ to provide a global correction law:

$$
\left\{\begin{array}{l}
T=a_{0}+a_{1} T_{a p p}+a_{2} T_{a p p}{ }^{2}+a_{3} T_{a p p}{ }^{3} \\
a_{i}=b_{i, 0}+b_{i, 1} h+b_{i, 2} \frac{h}{\cos \theta} \quad i=0,3
\end{array}\right.
$$

The residual error for the retrieved temperature is plotted in fig. 10-right. It is essentially less than $0.02^{\circ} \mathrm{C}$ when altitude variations are restricted to $[1000 \mathrm{~m}-1180 \mathrm{~m}]$ and when the viewing angle is restricted to $+/-30^{\circ}$ around nadir. Let us mention that a 0.01 emissivity estimation error induces a much higher error, namely between $0.411^{\circ} \mathrm{and} 0.55^{\circ} \mathrm{C}$ for a true temperature between $10^{\circ} \mathrm{C}$ and $30^{\circ} \mathrm{C}$.
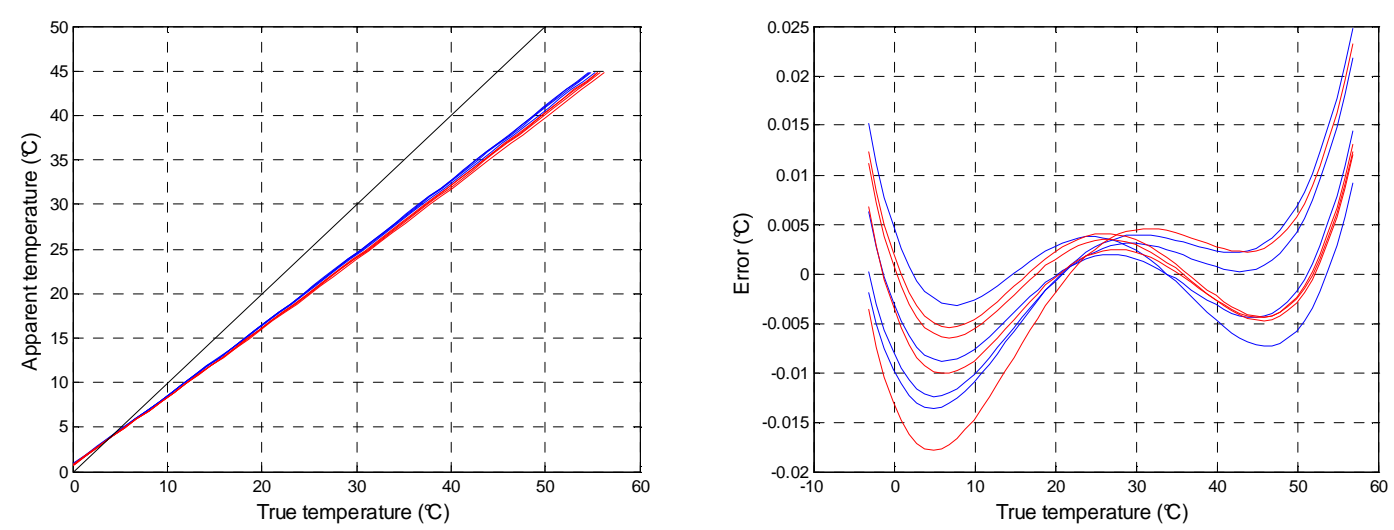

Fig. 10. Left: apparent temperature vs. true temperature depending on the sensor altitude and on the viewing angle. Altitude is $1000 \mathrm{~m}$ for the blue curves and $1180 \mathrm{~m}$ for the red ones. In each bundle the viewing angle ranges from $180^{\circ}$ (nadir) to $150^{\circ}$. Left : Residual error when th e true temperature is retrieved from the apparent temperature through the polynomial regression law described in Eq. (6).

\section{Geolocation of the remote sensing data}

GPS sensor joint with IMU provided the vehicle body coordinates frame evolution at $50 \mathrm{~Hz}$ frequency. Transformation of the infrared images in the camera frame to the body frame required a precise evaluation of the rotation and translation between body frame and camera frame (the IMU was in the left pod with the radar sensor whereas the IR camera was in the right pod). For this purpose, successive images containing a series of easily distinguishable control points were analysed (each control point could be followed in 3 to 11 successive images). The unknown parameters were identified by minimizing the scattering of the retrieved at-ground coordinates for each control point. The mean residual standard error was $5.0 \mathrm{~m}$. This error will be reduced in the future by installing an IMU in the same pod as the IR camera.

Brightness temperature for each pixel was projected on a UTM map by taking into account a Digital Elevation Model of the region. Motorglider height, pith and roll were also used for performing the atmosphere correction as described in $\S 3$. Thanks to the important overlapping rate of successive images, just $20 \%$ of the height of each image height was considered for the mozaicking.

Five flight lines were necessary for covering the region of interest. Each line was flown in only 60s, however about 21 min separated the fist and the last line (at 10:04 and 10:25 local time, respectively). Soil temperature evolved rather rapidly during this period of the morning which induced a noticeable contrast between the lines in the global image. A specific flux-based type correction was devised for eliminating this artefact. Fig. 11-left shows the final result. The lines now join with a barely detectable stitch. 
The corresponding NDVI image is reported in fig. 11-right. At fist glance, fields with high NDVI values (forests, orchards, high crops) show a low temperature. As a matter of fact surface temperature of canopy is closer to air temperature, which was about $10^{\circ} \mathrm{C}$, than temperature of bare soil which has already heated up since sunrise.

A preliminary analysis of the relation between temperature, vegetation index and soil moisture was performed with the results extracted from the maps in fig. 11 at the coordinates corresponding to the sixteen ground FDR measurements. The 3D plot is reported in fig. 12. A different color was used for each on-site measurement. The scatter observed for each color was artificially obtained by adding a random noise to all three variables in correspondence to the respective measurement variability. The classical anticorrelation between temperature and soil moisture is generally observed for all vegetation cover fraction values. The only exception is for the black dots which correspond to a dripirrigated orchard with young peach trees: moisture is high, about $25 \%$ wt, NDVI is relatively high, about 0.7 , however temperature is higher than expected, between 13.7 and $15^{\circ} \mathrm{C}$.
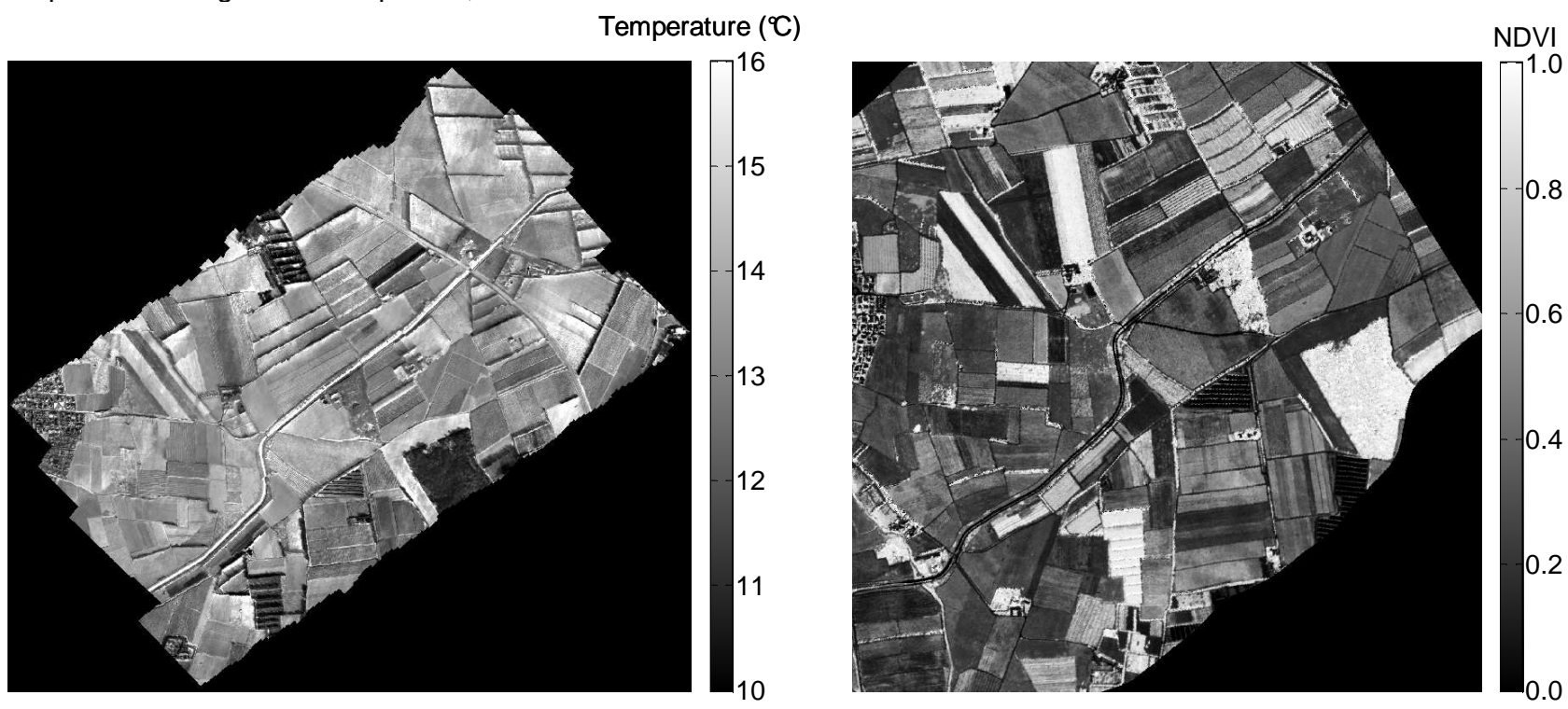

Fig. 11. Left : Temperature map obtained after orthorectification and flux normalization of 448 IR images recorded along five flight lines. Agriculture fields near Garons, France, Oct. $21^{\text {st }}$, 2011. Right : NDVI image obtained with the Hyspex camera of the same region, Oct, $5^{\text {th }}$

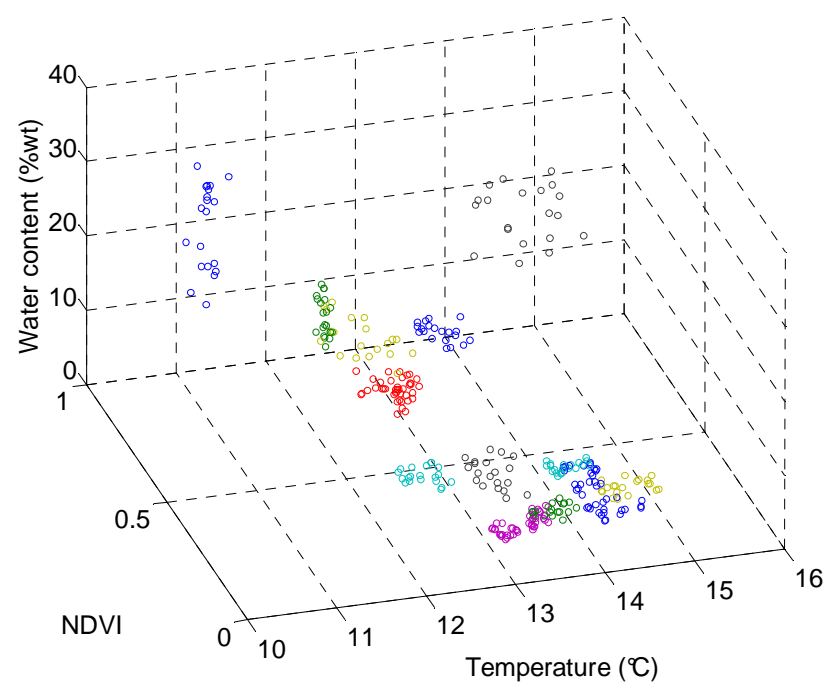

Fig. 12. 3D plot of temperature, NDVI and soil moisture. Temperature and NDVI are remote sensing data; soil moisture was obtained on-site with a FDR probe. 


\section{Conclusion}

A remote sensing measurement campaign was organized for validating a series of concepts, among which the possibility of mapping the soil moisture content from optical data in the red band, near infrared band and thermal infrared band. The thermal camera was of microbolometer type. Its angular resolution and its radiometric sensitivity were high enough for present application. Specific procedures were developed for performing a precise atmospheric compensation and an orthorectification of IR images recorded during temperature evolving conditions. Better results regarding the orthorectification process will be obtained in the future by using an IMU close to the camera. Additional work is needed for establishing a calibration relation for inferring soil moisture from radiative temperature and vegetation index. An experiment over a region encompassing areas where soil moisture is monitored simultaneously at different depths and over a broad range is of prime importance for making this calibration robust enough.

\section{Acknowledgements}

This research is within the framework of ONERA project ENVIRO.

\section{REFERENCES}

[1] Moran M.S., Clarke T.R., Inoue Y., Vidal A., "Estimating Crop water deficit using the relation between surfaceair temperature and spectral vegetation index", Remote Sensing of Environment 49, 246-263 (1994).

[2] Carlson T.N., Gillies R.R., Schmugge T. G., "An interpretation of methodologies for indirect measurement of soil water content", Agricultural and Forest Meteorology 77, 191-205 (1995).

[3] Carlson T.N., "An overview of the triangle method for estimating surface evapotranspiration and soil moisture from satellite imagery", Sensors, 7, pp. 1612-1629 (2007).

[4] Sandholt I., Rasmussen K., Andersen J., "A simple interpretation of the surface temperature-vegetation index space for assessment of surface moisture status", Remote Sensing of Environment 79, 213-224 (2002).

[5] Krapez J.-C., Olioso A., Coudert B., "Comparison of three methods based on the temperature-NDVI diagram for soil moisture characterization", Proc. of SPIE 7472, 74720y, 1-12 (2009).

[6] Rondeaux G., Steven M., Baret F., "Optimization of soil adjusted vegetation indices", Remote Sensing of Environment, vol. 55, 95-107, 1996.

[7] Maltese A., Cammalleri C., Capodici F., Ciraolo G., la Loggia G., "Surface soil humidity retrieval using remote sensing techniques: a triangle method validation", Proc. of SPIE 7824, 782425, 1-8 (2010).

[8] Krapez J.-C., Olioso A., " Improvement of the Triangle method for soil moisture evaluation by adding a third index: albedo or Cellulose Absorption Index", ", Proc. of SPIE 8174, 81740L-1-12 (2011).

[9] Tang R., Li Z.L., Tang B., "An application of the Ts-VI triangle method with enhanced edges determination for evapotranspiration estimation from MODIS data in arid and semi-arid regions: implementation and validation", Remote Sensing of Environment 114(3), 540-551 (2010).

[10] Vincente-Serrano S.M., Pons-Fernandez X., Cuadrat-Prats J.M., "Mapping soil moisture in the central Ebro river valley with Landsat and NOAA satellite imagery: a comparison with meteorological data", Int. J. Remote Sensing 25(20), 4325-4350 (2004).

[11] Chauhan N.S., Miller S., Ardanuy P., "Spaceborne soil moisture estimation at high resolution: a microwaveoptical/IR synergistic approach", Int. J. Remote Sensing 24(22), 4599-4622 (2003).

[12] Sobrino J.A., Franch B., Mattar C., Jimenez-Munoz J.C., Corbari C., "A method to estimate soil moisture from Airborne Hyperspectral Scanner (AHS) and ASTER data : Application to SEN2FLEX and SEN3EXP campaigns", Remote Sensing of Environment 117, 415-428 (2012).

[13] Baldridge A. M., Hook S. J., Grove C. I., Rivera G., "The ASTER Spectral Library Version 2.0", Remote Sensing of Environment 113, p.711-715, http://speclib.jpl.nasa.gov/, (2009).

[14] Fauqueux S., Labarre L., Caillault K., Malherbe C., Roblin A., Rosier B., Simoneau P., "MATISSE-v2.0, a radiative transfer code for advanced infrared earth modelling.", OPTRO 2012, ONERA TP 2012-497 (2012).

[15] Krapez J.-C., in: "Thermal measurements and inverse techniques", Chap. 6, H.R.B. Orlande et al. eds., CRC Press, 2011. 